\section{RMD Open}

Rheumatic \&

Musculoskeletal Diseases

\title{
Fibrocytes in early and long-standing rheumatoid arthritis: a 6-month trial with repeated synovial biopsy, imaging and lung function test
}

To cite: Just SA, Nielsen C, Werlinrud JC, et al. Fibrocytes in early and long-standing rheumatoid arthritis: a 6-month trial with repeated synovial biopsy, imaging and lung function test. RMD Open 2021;7:e001494. doi:10.1136/ rmdopen-2020-001494

- Additional material is published online only. To view please visit the journal online (http://dx.doi.org/10.1136/ rmdopen-2020-001494).

Received 25 0ctober 2020 Revised 1 February 2021 Accepted 22 February 2021

Check for updates

\section{(c) Author(s) (or their} employer(s)) 2021. Re-use permitted under CC BY-NC. No commercial re-use. See rights and permissions. Published by BMJ.

For numbered affiliations see end of article.

\section{Correspondence to} Dr Søren Andreas Just; soeren.andreas.just@rsyd.dk

\section{ABSTRACT}

Objectives To correlate the level of fibrocytes in peripheral blood, synovial tissue and in vitro culture in rheumatoid arthritis (RA) with changes in disease activity, imaging and pulmonary function.

Methods Twenty patients with early RA (ERA) and 20 patients with long-standing RA (LRA) were enrolled in a 6-month prospective study. Sixteen patients undergoing wrist arthroscopy were healthy controls. Patients with RA underwent pulmonary function tests, ultrasound and synovial ultrasound-guided needle biopsy of the same wrist at baseline and 6 months. Wrist MRI was performed at baseline (all) and 6 months (ERA). Circulating fibrocytes were measured by flow cytometry, in vitro by the number of monocytes that were differentiated to fibrocytes and in synovial biopsies by counting in histological sections.

Results Fibrocytes were primarily located around vessels and in the subintimal area in the synovium. Fibrocyte levels did not decline during the trial despite effective $R A$ treatment. In the ERA group, increased synovitis assessed by ultrasound was moderate and strongly correlated with an increase in circulating and synovial fibrocyte levels, respectively. Increased synovitis assessed by MRI during the trial in the ERA group was moderately correlated with both increased numbers of circulating and cultured fibrocytes. Absolute diffusion capacity level was overall weakly negatively correlated with the level of circulating and synovial fibrocytes. The decline in diffusion capacity during the trial was moderately correlated with increased levels of synovial fibrocytes.

Conclusion Our findings suggest that fibrocytes are involved in RA pathogenesis, both in the synovium and the reduction in lung function seen in a part of patients with RA.

Trial registration number NCT02652299.

\section{INTRODUCTION}

Fibrocytes are bone marrow-derived cells that express both haematopoietic (eg, CD $45^{+}$and $\mathrm{CD}^{+} 4^{+}$) and stromal comarkers (eg, collagen

\section{Key messages}

What is already known about this subject?

- Fibrocyte levels are correlated with interstitial lung disease (ILD) progression, and in murine arthritis, models have been found to home to inflamed joints and enhance arthritis activity.

What does this study add?

- Both early RA and long-standing RA have higher levels of circulating and synovial fibrocytes than healthy control.

- Fibrocytes were primarily located around vessels and in the subintimal area in the synovium.

- Rising levels of synovial fibrocytes during the trial were moderately correlated with a reduction in both forced ventilatory capacity and haemoglobin corrected diffusion capacity for carbon monoxide.

How might this impact on clinical practice?

- Fibrocytes could be a pathogenic link between joint and RA lung pathology, suggesting the new therapies targeting fibrocytes as a path forward in RA RA-ILD treatment.

and $\alpha$-smooth muscle actin $(\alpha$-SMA $)) .{ }^{1}$ In murine collagen-induced arthritis models, circulating fibrocytes have been found to home to inflamed joints and enhance arthritis activity. $^{2} 3$ The cell has, therefore, been proposed to be precursor cells for the fibroblast-like synoviocytes, which is central in the arthritis pathogenesis. ${ }^{2-7}$ In murine models, fibrocytes have been found to be located around vessels and in the synovial lining in the synovial tissue. ${ }^{289}$

After entering the inflamed tissue, the circulating fibrocytes differentiate into mature fibrocytes, which have a characteristic spindle shape (see figure 1A,B) and produce 


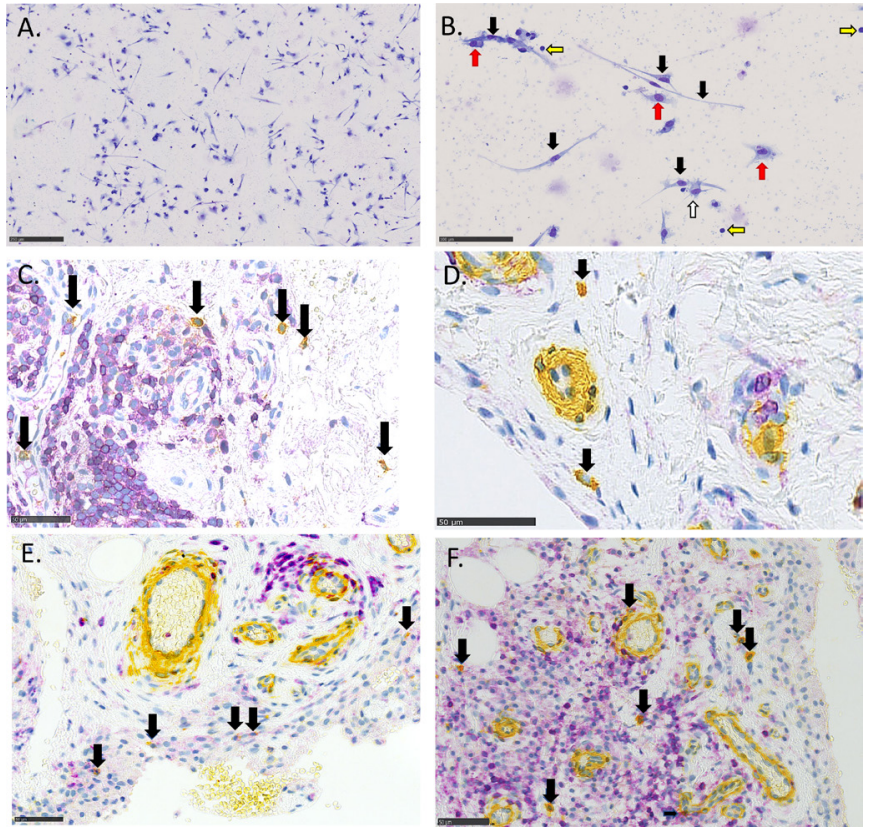

Figure 1 Fibrocytes in in vitro culture (A and $B$ ) and in the synovium (C, D, E and F). (A and B) PBMCs cultured for 5 days with interleukin-4. (B) Higher magnification, with black arrows marking fibrocytes, red arrows marking macrophages, white marking a dendritic cell and yellow marking lymphocytes. (C, D, E and F) Synovial tissue stained with CD45 (purple) and $\alpha$-smooth muscle actin (yellow), double-positive cells (fibrocytes) with dark arrows. (D) With low-grade inflammation and (C, E and F) with severe inflammation showing a high influx of lymphocytes around the vessels.

extracellular matrix proteins. ${ }^{2}{ }^{8}$ Fibrocyte differentiation is stimulated by both fibroblasts by means of lumican, $\mathrm{T}$ helper $2\left(\mathrm{~T}_{\mathrm{H}} 2\right)$ cells by means of cytokines interleukin (IL)-4 and IL-13, and is inhibited by T helper $1\left(\mathrm{~T}_{\mathrm{H}} 1\right)$ cytokines and serum amyloid P (SAP). ${ }^{11}$

Fibrocyte levels are correlated with disease progression and mortality in interstitial lung disease (ILD) and are identified as a new treatment target. ${ }^{12-14}$ ILD can also be an extra-articular manifestation of RA (RAILD), leading to a reduced lung diffusion capacity and increased mortality. ${ }^{15}$ An influx of fibrocytes in diseased organs is a characteristic of fibrotic diseases. ${ }^{716}$ In animal pulmonary fibrosis models, the levels of circulating fibrocytes have been shown to correlate with increased lung collagen deposition and fibrosis. ${ }^{17} 18$ Previously, we have shown that circulating fibrocyte levels are elevated in RA, RA-ILD, severe asthma and fibrotic ILDs when compared with healthy controls (HCs). ${ }^{19}$

To study how levels of circulating in vitro differentiated fibrocytes and synovial tissue-resident fibrocytes are related to RA disease activity, joint imaging, synovial histopathology and lung function, we, therefore, designed a novel prospective exploratory study of both early treatment-naive (ERA) and long-standing RA (LRA) patients followed up for 6 months.

\section{PATIENTS AND METHODS}

\section{Study design}

A prospective longitudinal study (baseline, 3 months and 6 months visit) was conducted at Odense University Hospital (OUH), Denmark (ClinicalTrials.gov: NCT02652299). All patients were recruited following written informed consent, and the study was reviewed by the regional ethics review board (S-20140062) and the Danish Data Protection Agency (2008-58-0035).

Data from this cohort comparing wrist synovial biopsy inflammation with disease activity in the wrist by X-ray, ultrasound and MRI have been published separately. ${ }^{20}$ Overlapping results are a description of ultrasound and MRI data, synovial inflammation and RA disease activity. In this article, we use the Larsen score for all joints of the biopsied hand and not only the biopsied wrist.

\section{Patients}

Patients were enrolled from the Department of Medicine, Section of Rheumatology, Svendborg Hospital OUH, and Department of Rheumatology, OUH, and Department of Orthopaedic Surgery, OUH. Forty-three patients with RA were assessed for eligibility, and three were excluded (two had Calcium Pyrophosphate Depostion disease (CPPD) and one Polymyalgia Rheumatica). All patients fulfilled the ACR/EULAR 2010 RA classification criteria and had at least one swollen wrist. The ERA group $(n=20)$ were newly diagnosed and treatment-naive, while the LRA patients had disease duration over five years. RA patients were treated during the study according to treatment guidelines from the Danish Rheumatological Association. ${ }^{21}$ Patients in the control group underwent wrist arthroscopy at the Departmentof Orthopedic Surgery, OUH, due to mild osteoarthrosis or ganglion, and had no inflammatory joint diseases or signs of RA in baseline clinical examination or blood samples. A total of 17 patients were enrolled, one was excluded as synovialbiopsies were not taken during arthroscopy. The planned 20 controls could not be reached before the set date for the end of inclusion.

Patient demographics and rheumatological medication (disease-modifying antirheumatic drugs (DMARDs), biologicals, corticosteroid), Disease Activity Score in 28 joints with C-reactive protein (DAS28CRP) were collected at baseline, 3 months and 6 months.

\section{Synovial biopsies}

At baseline and 6 months visit, ultrasound-guided needle biopsy (USNB) was performed in a clean procedure room, as previously described.$^{22}$ Biopsies were obtained from the same wrist at baseline and 6 months. See online supplemental materials methodsfor details on the USNB procedure. The degree of inflammation and cell infiltration was determined by SAJ and an experienced pathologist (HDS) using the semiquantitative Krenn score and semiquantitative scores (0-4) as previously described. ${ }^{23}$ 


\section{Fibrocyte quantification}

\section{Circulating fibrocytes}

The number of circulating fibrocytes $\left(\mathrm{CD} 45^{+} \mathrm{CD} 34^{+}\right.$

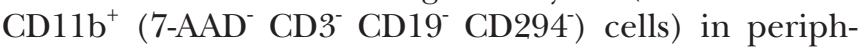
eral blood was measured by flow cytometry, a protocol we have previously described and validated (online supplemental materials methods). ${ }^{19}$

\section{In vitro differentiated fibrocytes}

Peripheral blood mononuclear cells (PBMCs) were cultured for 5 days to determine the number of differentiated fibrocytes per $10^{5}$ cultured monocytes, as previously described (online supplemental materials methods). ${ }^{24} 25$ The quantification of fibrocytes was done by microscopy. Mature fibrocytes were defined as spindle-shaped cells with a minimum length of $40 \mu \mathrm{m}$ (see figure 1A,B).

\section{Synovial fibrocytes}

Three micrometre sections of paraffin-embedded synovial biopsies were double-stained using immunohistochemistry and antibodies for CD45 and $\alpha$-SMA, and double-positive cells were defined as tissue-resident fibrocytes (figure 1C-F and online supplemental materials methods) ${ }^{26}$ The combination of markers has previously been used for studying fibrocytes in various tissues. ${ }^{3} 2728$

\section{Radiological assessment}

X-ray of hands, feet and chest was conducted at baseline and 6 months. Radiographic data from the biopsied hand were scored by a consultant in radiology (TT) using Larsen score to evaluate radiographic progression. ${ }^{29}$ On the same side as the USGSB procedure was to be performed, an MRI of the hand and wrist was performed, as previously described. ${ }^{20}$ MRI was performed for a maximum of 2 hours before the biopsy procedure for all at baseline and also at 6 months for ERA. Using the RAMRIS system, a consultant in radiology (TT) scored all examinations. Ultrasound of the hand and wrist undergoing synovial biopsy was performed on all visits (baseline, 3 months and 6 months) according to the EULAR OMERACT (EO) scoring system (see the online supplemental materials methods section). All patients underwent chest X-ray at baseline and 6 months, and if described with fibrosis, the results were registered.

\section{Lung physiology}

Pulmonary function tests were obtained at baseline and after 6 months. Over the study period, a reduction of $5 \%$ or more of forced ventilatory capacity (FVC) or haemoglobin corrected diffusion capacity for carbon monoxide $\left(\mathrm{DLCO}_{c}\right)$ was defined as clinically significant, following guidelines. ${ }^{30} 31$ A pathological reduced $\mathrm{DLCO}_{c}$ was defined as $80 \%$ of predicted value or below.

Patients with reduced diffusion capacity were referred to pulmonary medicine for further investigation, including high-resolution CT (HRCT) if deemed clinically appropriate.

\section{Statistics}

Categorical variables were presented as numbers and percentages and continuous variables as means and SD. One-way analysis of variance (for continuous variables) or Fisher's exact (categorical variables) test was used to compare variables between groups in baseline data. Linear regression with cluster robust SEs was used to test for trend in each patient group. Robust cluster estimation was used in the linear regressions to account for minor deviations from the model assumptions on normality and to account for repeated measurements of patients. Comparisons of RA groups with HC were performed using unadjusted linear regression. Unadjusted logistic regression was used to test if the level of fibrocytes was a risk factor for FVC and $\mathrm{DLCO}_{c}$ reduction over 6 months. Pairwise Pearson correlations between DAS28CRP, synovial inflammation, imaging scores, fibrocytes and lung function at baseline and 6 months, and for change of measurements during the trial, were calculated. $P$ values $<0.05$ were considered as statistically significant. Data were analysed on Stata V.16 (StataCorp, Texas, USA).

\section{RESULTS}

\section{Patients and procedures}

Patients and disease characteristics of the 56 patients are presented in table 1 .

The mean age was $57( \pm 13.7)$ years, with significantly lower age in the HC group versus all RA $(p<0.01)$, although with no age difference between ERA and LRA $(p=0.19)$. A majority of female patients was found in all groups with no difference between groups $(p=0.58)$. There was no difference in smoking status between ERA and LRA groups $(\mathrm{p}=0.37)$. None of the HCs was CCP or $\mathrm{RF}$ positive, and there was no difference between the fraction of seropositive (CCP or RF) between the ERA group and the LRA group $(p=0.49)$. ERA disease duration was 0.06 year $( \pm 0.14)$ and 13.6 years $( \pm 8.46)$ in the LRA group. The majority $(n=16)$ of LRA patients were on methotrexate treatment (alone or in combination with other therapies) (for details, see online supplemental table 1). There was no difference in $\mathrm{DLCO}_{c}(\%$ of predicted) at baseline between LRA and ERA ( $p=0.59)$, while the FVC (\% of predicted) was higher in the ERA group $(\mathrm{p}=0.04)$. There was higher DAS28CRP disease activity in the ERA group $(<0.01)$, with a more erosive disease in the LRA group $(\mathrm{p}=0.02)$. Baseline chest $\mathrm{X}$-ray showed signs of fibrosis in 3 out of $20(16 \%)$ in ERA versus 0 out of $20(0 \%)$ in the LRA group.

\section{Change in disease activity, inflammation on imaging, pulmonary function and fibrocyte levels}

We next evaluated changes in disease activity, pulmonary function, imaging inflammation scores, synovial inflammation and numbers of peripheral and cultured fibrocytes during the study (table 2).

RA disease activity decreased in both the ERA and the LRA groups (both $\mathrm{p}<0.001$ ), while CRP concentrations 


\begin{tabular}{|c|c|c|c|c|}
\hline Groups & ERA & LRA & $\mathrm{HC}$ & $P$ values \\
\hline Patients, n (\%) & $20(36)$ & $20(36)$ & $16(28)$ & \\
\hline Age & $64.5(8.9)$ & $60.8(8.5)$ & $43.8(14.1)$ & $<0.01$ \\
\hline Female, n (\%) & $15(75)$ & $12(60)$ & $12(71)$ & 0.58 \\
\hline Current/former/never smoker & $32 \% / 32 \% / 36$ & $16 \% / 26 \% / 58 \%$ & & 0.37 \\
\hline \multicolumn{5}{|l|}{ Blood sample results } \\
\hline CRP, mg/L & $20.4(19.8)$ & 15.9 (18.9) & $1.8(2.3)$ & $<0.01$ \\
\hline Monocytes, $10^{9} / \mathrm{L}$ & $0.62(0.3)$ & $0.65(0.2)$ & $0.6(0.2)$ & 0.73 \\
\hline RF or CCP-positive, n (\%) & $14(64)$ & $15(71)$ & $0(0)$ & $<0.01$ \\
\hline \multicolumn{5}{|l|}{ Pulmonary function test } \\
\hline $\mathrm{FEV}_{1}(\%$ predicted $)$ & $111.5(25)$ & $102.7(17)$ & & 0.21 \\
\hline FVC (\% predicted) & $122.5(23)$ & $108.8(16)$ & & 0.04 \\
\hline TLC (\% predicted) & $112(18)$ & $104.5(17)$ & & 0.30 \\
\hline $\mathrm{DLCO}_{\mathrm{c}}(\%$ predicted $)$ & $90.8(17)$ & $92.0(10)$ & & 0.79 \\
\hline \multicolumn{5}{|l|}{ Rheumatoid arthritis characteristics } \\
\hline Disease duration (years) & $0.06(0.14)$ & $13.58(8.46)$ & & $<0.01$ \\
\hline DAS28CRP & $5.45(1.30)$ & $4.25(0.85)$ & & $<0.01$ \\
\hline \multicolumn{5}{|l|}{ EULAR disease activity } \\
\hline Remission, n (\%) & $0(0)$ & $0(0)$ & & \\
\hline Low activity, $\mathrm{n}(\%)$ & $0(0)$ & $0(0)$ & & \\
\hline Moderate activity, n (\%) & $6(30)$ & $18(90)$ & & \\
\hline High activity, $\mathrm{n}(\%)$ & $14(70)$ & $2(10)$ & & $<0.01$ \\
\hline Erosive disease $^{\star}$ & $3(15.8)$ & $10(50)$ & & 0.02 \\
\hline \multicolumn{5}{|l|}{ Treatment } \\
\hline Naive/Not in treatment, $\mathrm{n}(\%)$ & $20(100.0)$ & $2(10.0)$ & & \\
\hline cDMARD, n (\%) & $0(0.0)$ & $17(45.0)$ & & \\
\hline bDMARD, n (\%) & $0(0.0)$ & $2(10.0)$ & & $<0.01$ \\
\hline Peroral steroid, n (\%) & $0(0.0)$ & $1(5.0)$ & & 0.31 \\
\hline
\end{tabular}

*Defined as erosions on hands or feet X-ray compatible with RA damage.

bDMARD, biological disease-modifying antirheumatic drug; CCP, cyclic citrullinated peptide; cDMARD, conventional disease-modifying antirheumatic drug; CRP, C-reactive protein; DAS28CRP, Disease Activity Score 28 joints combined with CRP value; DLCOc, diffusion

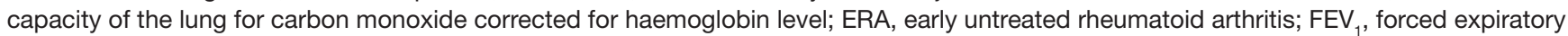
volume in $1 \mathrm{~s}$; FVC, forced ventilatory capacity; HC, healthy control; LRA, long-standing rheumatoid arthritis; RF, rheumatoid factor; TLC, total lung capacity.

decreased in the ERA but not in the LRA group. X-ray combined Larsen score of the hand did not change during the study period in either ERA or LRA. The radiocarpal joint assessed by the RAMRIS scoring system showed declining synovitis in the ERA group, but unchanged bone marrow oedema and erosions scores during the trial. Radiocarpal intercarpal inflammation assessed by ultrasound did not change in the combined score during the trial, but the colour Doppler score declined in both groups. Wrist synovial biopsy inflammation was higher in both ERA and LRA than HC and decreased significantly in ERA but not in LRA. For the change in synovial tissue levels of CD20, CD68, CD3 and CD138, see online supplemental table 2.

Baseline levels of all fibrocyte measurements were significantly higher in both ERA and LRA compared with HC, except for cultured fibrocytes in the ERA group (table 2). There was no difference in the baseline level of circulating or synovial fibrocytes between the ERA and LRA groups ( $\mathrm{p}=0.65$ and $\mathrm{p}=0.21$, respectively). Baseline cultured fibrocyte levels were higher in LRA than ERA $(p=0.01)$. Circulating fibrocytes were unchanged during the study in both groups (table 2). Overall, 23\% $(32 / 136)$ of all fibrocyte cell cultures failed (ERA: $22 \%$ $(13 / 60)$, LRA $25 \%(15 / 60)$ and HC $25 \%(4 / 16))$, with no significant difference between groups. The numbers of cultured fibrocytes were unchanged during the study period in both RA groups $(\mathrm{p}=0.24$ and $\mathrm{p}=0.08$, respectively) (table 2). Synovial inflammation decreased in the ERA group but not in the LRA group.

FVC and DLCO $_{c}$ (\% of predicted) values were overall unchanged during the study in both RA groups. At 
Table 2 Disease activity, imaging, lung function, biopsy inflammation and fibrocyte levels

\begin{tabular}{|c|c|c|c|c|c|c|}
\hline & \multicolumn{3}{|l|}{ ERA } & \multicolumn{3}{|c|}{$\mathrm{HC}$} \\
\hline & $\begin{array}{l}\text { Visit } \\
\text { at baseline }\end{array}$ & $\begin{array}{l}\text { Visit } \\
\text { at } 3 \text { months }\end{array}$ & $\begin{array}{l}\text { Visit } \\
\text { at } 6 \text { months }\end{array}$ & $\begin{array}{l}\text { Test for } \\
\text { trend, } p\end{array}$ & Baseline & $\begin{array}{l}P \\
\text { values }^{*}\end{array}$ \\
\hline$n$ & 20 & 19 & 20 & & 16 & \\
\hline DAS28CRP & $5.2(1.0)$ & $2.9(1.2)$ & $2.6(1.12)$ & $<0.001$ & & \\
\hline CRP & $20.4(19.8)$ & $12.0(13.7)$ & $8.7(12.8)$ & 0.01 & $1.77(2.2)$ & 0.001 \\
\hline Hand Larsen score† & $0.65(1.4)$ & & $1.00(1.63)$ & 0.09 & $0.0(0.0)$ & 0.05 \\
\hline \multicolumn{7}{|l|}{ Pulmonary function test $\ddagger$} \\
\hline $\mathrm{FEV}_{1}(\%$ predicted $)$ & $111(25)$ & & $109(21)$ & 0.36 & & \\
\hline FVC (\% predicted) & $122(23)$ & & $120(19)$ & 0.40 & & \\
\hline TLC (\% predicted) & $112(18)$ & & $110(18)$ & 0.38 & & \\
\hline $\mathrm{DLCO}_{\mathrm{c}}(\%$ predicted $)$ & $91(16)$ & & $88(14)$ & 0.08 & & \\
\hline \multicolumn{7}{|c|}{ MRI RAMRIS score, Radiocarpal joint§ } \\
\hline Synovitis $(0-3)^{\star \star}$ & $1.15(0.93)$ & & $0.80(0.52)$ & 0.02 & $0.30(0.48)$ & 0.003 \\
\hline Bone erosions $(0-10)$ & $0.15(0.37)$ & & $0.15(0.37)$ & 1.00 & $0.00(0.00)$ & 0.08 \\
\hline Bone oedema (0-3) & $0.11(0.32)$ & & $0.05(0.22)$ & 0.31 & $0.00(0.00)$ & 0.16 \\
\hline \multicolumn{7}{|c|}{ US EULAR-OMERACT, Radiocarpal joints } \\
\hline Combined score (0-3) & $2.1(0.8)$ & $1.9(0.7)$ & $1.6(0.6)$ & 0.07 & & \\
\hline SH score (0-3) & $1.9(0.8)$ & $1.9(0.7)$ & $1.6(0.6)$ & 0.17 & & \\
\hline CD score $(0-3)$ & $1.7(1.0)$ & $1.2(0.9)$ & $1.0(0.7)$ & 0.001 & & \\
\hline \multicolumn{7}{|c|}{ Synovial biopsy from Radiocarpal joint } \\
\hline Inflammation score (Krenn) & $6.0(1.5)$ & & $5.0(1.4)$ & 0.01 & $4.2(1.7)$ & 0.002 \\
\hline \multicolumn{7}{|l|}{ Circulating fibrocytes } \\
\hline $\mathrm{CD}_{4} 5^{+} \mathrm{CD} 34^{+} \mathrm{CD} 11 \mathrm{~b}^{+} / \mu \mathrm{L}$ & $7.2(3.3)$ & $6.0(2.0)$ & $5.5(2.0)$ & 0.08 & $4.0(1.8)$ & 0.001 \\
\hline \multicolumn{7}{|l|}{ Cultured fibrocytes \{} \\
\hline Fibrocytes $/ 10^{5}$ monocytes & $3503(2097)$ & $3334(1453)$ & $6313(4666)$ & 0.08 & $2522(2218)$ & 0.24 \\
\hline \multicolumn{7}{|l|}{ Synovial fibrocytes } \\
\hline \multirow[t]{2}{*}{ Fibrocytes $/ \mathrm{mm}^{2}$} & $39.7(17)$ & & $39.5(18)$ & 0.95 & $22.4(12.3)$ & 0.001 \\
\hline & LRA & & & & $\mathrm{HC}$ & \\
\hline $\mathrm{n}$ & 20 & 20 & 20 & & 16 & \\
\hline DAS28CRP & $4.26(0.64)$ & $3.17(1.04)$ & $2.56(1.05)$ & $<0.001$ & & \\
\hline CRP & $15.6(18.9)$ & $10.3(11.4)$ & $10.3(16.3)$ & 0.08 & $1.77(2.2)$ & 0.002 \\
\hline Hand Larsen score $†$ & $8.55(9.8)$ & & $8.68(10.0)$ & 0.46 & $0.0(0.0)$ & 0.001 \\
\hline \multicolumn{7}{|l|}{ Pulmonary function test $\ddagger$} \\
\hline $\mathrm{FEV}_{1}(\%$ predicted) & $102(17)$ & & $102(21)$ & 0.89 & & \\
\hline FVC (\% predicted) & $109(16)$ & & $109(17)$ & 0.75 & & \\
\hline TLC (\% predicted) & $104(17)$ & & $103(16)$ & 0.67 & & \\
\hline $\mathrm{DLCO}_{\mathrm{c}}(\%$ predicted $)$ & $90(10)$ & & $88(12)$ & 0.22 & & \\
\hline \multicolumn{7}{|c|}{ MRI RAMRIS score, Radiocarpal joint § } \\
\hline Synovitis (0-3) & $1.95(1.23)$ & & & & $0.30(0.48)$ & $<0.001$ \\
\hline Bone erosions $(0-10)$ & $1.20(1.32)$ & & & & $0.00(0.00)$ & $<0.001$ \\
\hline Bone oedema $(0-3)$ & $0.30(0.73)$ & & & & $0.00(0.00)$ & 0.08 \\
\hline \multicolumn{7}{|c|}{ US EULAR-OMERACT, Radiocarpal joints } \\
\hline Combined score (0-3) & $2.3(0.8)$ & $2.1(0.9)$ & $1.9(0.8)$ & 0.17 & & \\
\hline $\mathrm{SH}(0-3)$ & $2.1(0.8)$ & $2.0(0.9)$ & $1.9(0.8)$ & 0.24 & & \\
\hline
\end{tabular}


Table 2 Continued

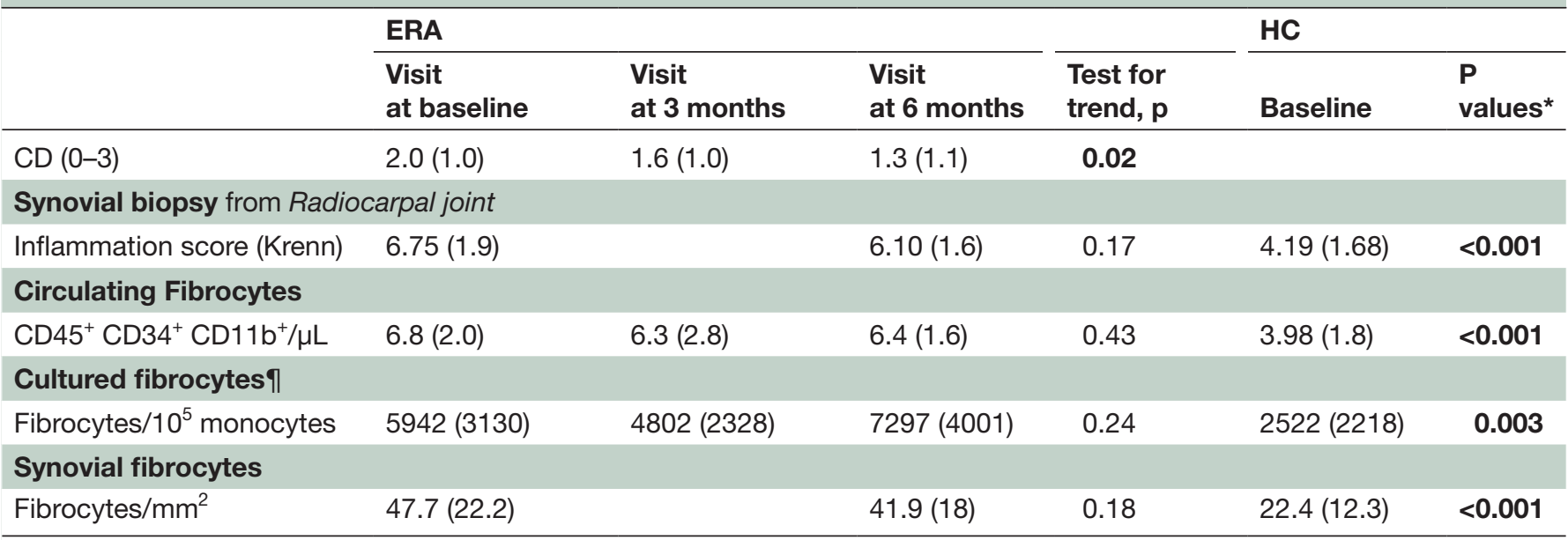

Mean (SD) unless otherwise indicated.

${ }^{*} P$ value control value vs respectively ERA and LRA baseline. Significant $p$ values in bold.

†Total Larsen score of the hand undergoing USNB.

†Four out of 80 (5\%) missing pulmonary function test, 2 in ERA and 2 in LRA.

$\S \mathrm{n}=6$ in $\mathrm{HC}$ had an MRI without contrast and $\mathrm{n}=1$ in ERA no MRI due to claustrophobia.

ๆOverall, 23\% of all fibrocyte cell cultures failed (ERA: 13/60, LRA 15/60, HC 4/16).

CD, colour Doppler; DAS28CRP, Disease Activity score in 28 joints with C-reactive Protein (CRP); ERA, early rheumatoid arthritis; LRA, long-

standing rheumatoid arthritis; $\mathrm{SH}$, synovial hypertrophy; USNB, ultrasound-guided needle biopsy.

baseline, 9 patients (6 ERA and 3 LRA) had reduced $\operatorname{DLCO}_{c}(\leq 80 \%$ of predicted) and at 6 months, it was 11 patients (7 ERA and 4 LRA). Fifteen patients had a significant reduction in $\mathrm{DLCO}_{c}(<5 \%)$ during the trial (LRA: 6, ERA: 9). Chest X-ray assessment did not change during the study (3/40 with fibrosis). Eight patients (20\%) underwent HRCT during the study (five ERA and three LRA), and four (10\%) had changes compatible with ILD (two ERA and two LRA). At the end of the trial, these four patients were followed up in a pulmonary ILD centre.

\section{Correlating circulating, cultured and synovial fibrocytes}

We found a moderate correlation between numbers of circulating and cultured fibrocytes (overall $\mathrm{r}=0.41$, $\mathrm{p}<0.001$, highest in $\mathrm{HC} r=0.62, \mathrm{p}=0.03$ ). The number of synovial fibrocytes was weakly correlated with circulating fibrocytes (overall $\mathrm{r}=0.25 \mathrm{p}=0.008$ ) but not with cultured fibrocytes (overall $\mathrm{r}=0.13, \mathrm{p}=0.19$ ). Detailed analyses of fibrocyte correlations in the RA subgroups at baseline and 6 months are shown in online supplemental table 3 .

\section{Fibrocytes and markers of RA disease activity}

Overall, we found no significant correlations between disease activity by DAS28CRP and circulating, cultured or synovial fibrocytes (online supplemental table 3 for the RA subgroups).

Overall, synovial fibrocyte levels were weakly correlated with the CRP level $(\mathrm{r}=0.23, \mathrm{p}=0.007)$, while cultured or circulating fibrocyte levels were not (data not shown). Erosion on X-ray was overall weakly correlated with the circulating fibrocyte level $(\mathrm{r}=0.20, \mathrm{p}=0.01)$, while cultured or synovial fibrocyte levels were not. Changes in CRP or Larsen score during the trial were not correlated with any change in fibrocyte measurements (data not shown).
Levels of fibrocytes in the synovium were overall moderately correlated with the $\mathrm{EO}$ combined in ultrasound score $(\mathrm{r}=0.38, \mathrm{p}=0.001)$, while circulating and cultured fibrocytes were not (see online supplemental table 3 for subgroup analyses). Change in ultrasound score during the trial was moderate to strongly correlated with change in circulating and cultured fibrocytes in the ERA group $(\mathrm{r}=0.47, \mathrm{p}=0.03$, and $\mathrm{r}=0.81, \mathrm{p}=0.001$ respectively $)$, while this was not seen in the LRA group (online supplemental table 4).

Change of MRI assessed synovitis during the trial in the ERA group was moderately correlated with changes in both circulating and cultured fibrocytes $(r=0.47$ and $\mathrm{r}=0.60$, respectively, both $\mathrm{p}=0.04$; online supplemental table 4).

Overall, synovial inflammation was moderately correlated with synovial fibrocytes $(\mathrm{r}=0.56, \mathrm{p}<0.001)$, weakly correlated with circulating fibrocytes $(\mathrm{r}=0.23$, $\mathrm{p}=0.02$ ) and not correlated with the level of cultured fibrocytes $(\mathrm{r}=0.18, \mathrm{p}=0.06)$ (online supplemental table 3 for subgroup analyses).

\section{Fibrocytes and RA lung function}

FVC ( $\%$ of predicted) values were weakly correlated with the number of cultured and synovial fibrocytes $(\mathrm{r}=-0.37$, $\mathrm{p}<0.001$ and $\mathrm{r}=-0.29, \mathrm{p}=0.04)$, but not with circulating fibrocytes $(\mathrm{r}=-0.16, \mathrm{p}=0.24)$ (online supplemental table 3 for subgroup analysis). The decline in FVC (\% of predicted) over the study period was moderately correlated with an increase in the number of synovial fibrocytes $(\mathrm{r}=-0.51 \mathrm{p}=0.001$, figure $2 \mathrm{D}$, see online supplemental table 3 for subgroups) but not with the number of circulating or cultured fibrocytes (data not shown). 


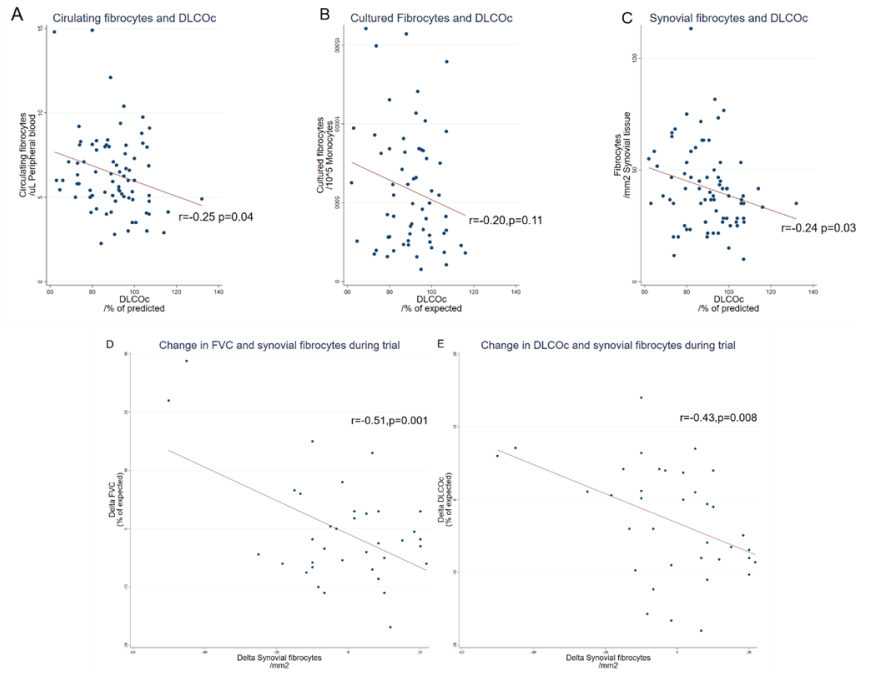

Figure 2 Fibrocytes and lung function. The absolute level of circulating (A), cultured (B) and synovial (C) fibrocytes correlated with diffusion capacity (\% of predicted). Change in forced ventilatory capacity (FVC) (D) and haemoglobin corrected diffusion capacity for carbon monoxide (DLCO $)(E)$ correlated with the change in synovial fibrocytes.

Overall, absolute $\mathrm{DLCO}_{\text {c }}$ (\% of predicted) value was weakly correlated with the levels of circulating and synovial fibrocytes, but not with the cultured fibrocytes (figure 2A-C, see online supplemental table 3 ). Change in $\mathrm{DLCO}_{\mathrm{c}}(\%$ of predicted) during the trial was moderately correlated with the rise in synovial fibrocytes (figure 2E), but not with the change in circulating or cultured fibrocytes (online supplemental table 4 ).

Baseline fibrocyte levels were not significantly different between RA patients with normal diffusion at 6 months versus those with reduced level $\left(\right.$ DLCO $\left._{c}>80 \%, n=11\right)$. At 6 months, we found higher levels of cultured fibrocytes (5978 vs 9429 fibrocytes $/ 10^{5}$ monocytes cultured, $\mathrm{p}=0.04)$ in patients with reduced diffusion capacity and circulating (5.6 vs 6.7 fibrocytes/ $\mu \mathrm{L}$ blood, $\mathrm{p}=0.047$ ), while no significant difference in synovial fibrocytes (42 vs $38 / \mathrm{mm}^{2}$ synovium, $\mathrm{p}=0.55$ ). In a subgroup analysis of the same population in the ERA group, the number of circulating fibrocytes were was at 6 months (4.8 vs 6.7 fibrocytes $/ \mu \mathrm{L}$ blood, $\mathrm{p}=0.049)$ in the reduced DLCO group, while not significant in the LRA group (6.3 vs 6.8 fibrocytes $/ \mu \mathrm{L}$ blood, $\mathrm{p}=0.55$ ). There was no difference in synovial fibrocyte level between the two groups, overall or in subgroup analysis (data not shown). Comparing the four patients with HRCT and lung function changes compatible with ILD with the remaining group, we did not find any difference in baseline fibrocyte levels. In the 6-month data, we saw a difference in circulating fibrocytes ( 7.5 vs 5.5 fibrocytes $/ \mu L$ blood, $\mathrm{p}=0.01$ ), but no difference in cultured or synovial fibrocytes (data not shown).

\section{Prediction of reduction in pulmonary function}

Baseline circulating, cultured or synovial fibrocytes could not predict a reduction in $\mathrm{DLCO}_{c}(\%$ of predicted) over
$5 \%$ or $\mathrm{DLCO}_{c}<80 \%$ at 6 months (neither could CRP, DAS28CRP or synovial inflammation) (data not shown).

\section{DISCUSSION}

This is the first prospective study investigating the role of fibrocytes in RA, showing (1) levels of circulating fibrocytes are moderately correlated with the number of monocytes that differentiate to fibrocytes in vitro in both RA and HCs, and weakly correlated with the number of fibrocytes in the synovium. (2) Both ERA and LRA have higher levels of circulating and synovial fibrocytes than HC. (3) Disease activity by DAS28CRP, or change over time, is not correlated with absolute numbers or change over time in any of the fibrocyte measurements. (4) Synovial fibrocytes are primarily located around vessels and in the subintimal area in the human synovium. (5) Synovial fibrocyte levels in RA are moderately correlated with disease activity in the biopsied joint assessed by ultrasound. (6) In the ERA group, change in joint inflammation evaluated by MRI and ultrasound is moderately correlated with the change in the level of both circulating and cultured fibrocytes. (7) Decline in FVC or $\mathrm{DLCO}_{c}$ was not correlated with the change in traditional RA disease biomarkers. (8) Overall, reduction in absolute DLCO (\% of predicted) was correlated with the rising levels of circulating and synovial fibrocytes. (9) Rising levels of synovial fibrocytes during the trial were moderately correlated with a reduction in both $\mathrm{FVC}$ and $\mathrm{DLCO}_{c}$. (10) Baseline fibrocyte levels could not predict pathological FVC or DLCO reduction at 6 months. (11) Patients with reduced $\mathrm{DLCO}_{c}$ at 6 months had a higher level of cultured fibrocytes, and in the ERA group also circulating fibrocytes compared with RA patients with normal DLCO .

This study is an explorative prospective study of a new possible RA biomarker and it has limitations. The cohort was relatively small, and treatment was not protocolled. Patients in the LRA group had different baseline treatments, according to national guidelines recommendations taking into account the course of previous arthritis activity. Therefore, the LRA group received a more variable treatment during trial compared with the ERA group, which could potentially affect outcome measures. A high fraction (23\%) of fibrocyte cultures failed. Protocols for measuring circulating fibrocytes traditionally include intracellular staining, for example, collagen or $\alpha$-SMA. However, protocols without intracellular staining, as applied here, have also been thoroughly validated. ${ }^{19}$ 32-34 The use of different methods for measuring circulating fibrocytes is a limitation for the comparability of results within fibrocyte research. HRCT was not part of the protocol. Multiple testing was used, so the results are to be interpreted with caution; we recommend the reader to focus on results significant at significance level of 0.01 . Using other marker combinations for staining synovial fibrocytes may result in a higher number of fibrocytes/ $\mathrm{mm}^{2}$, as shown in lung biopsies. ${ }^{35}$ 
Several of our findings point to fibrocytes as a key mediator of RA pathogenesis. In both RA groups, there was a significant reduction in disease activity, but still fibrocyte levels did not decline. Fibrocytes are located in the synovium, and quantitative change in both circulating and cultured fibrocytes is correlated with the change in ultrasound and MRI assessed inflammation. In murine arthritis models, fibrocytes infiltrate the synovium and thereby worsen arthritis activity. ${ }^{3} 8$ We thereby confirm previous findings in murine models. ${ }^{2}{ }^{3}$ Fibrocytes progressively lose their haematopoietic markers during differentiation, after entering target tissue. ${ }^{35-37}$ This is in line with the observed location of fibrocytes, close to the vessels, thereafter, gradually losing their haematopoietic markers. We show that patients with RA have a marked increase in infiltrating fibrocytes compared with HC. We, therefore, speculate that the cell could be involved in arthritis pathology, as it has recently been shown for synovial macrophages. ${ }^{38}$ The source of the increased number of circulating and synovial fibrocytes in RA remains to be established. Plausible models for increased number of circulating fibrocytes include increased proliferation, mobilisation and/or differentiation from bone marrow precursors, perhaps in the setting of systemic inflammation, or increased mobilisation from the excess number present in diseased synovium. ${ }^{716}$ The source of the synovial fibrocytes could be the increased differentiation from circulating fibrocytes and/or from the increased transition from other tissue-resident cells, for example, epithelial cells. ${ }^{735}$ Recently, it was described that 1-2 weeks before an RA flare, B-cell activation was followed by an increase of circulating preinflammatory mesenchymal cells (so-called PRIME cells) that share features with synovial fibroblasts. ${ }^{39}$ The authors propose that the PRIME cells are precursors to the inflammatory sublinning fibroblasts found adjacent to the vessels in the inflamed RA synovium. ${ }^{40}$ The finding supports a model where cells of the circulation migrate into the synovium and differentiate to pathogenic fibroblasts in the RA synovium. PRIME cells are not fibrocytes, as they are CD45, but fibrocytes could be a precursor cell for one of the other subgroups of pathogenic fibroblasts in the synovium. ${ }^{39} 40$

Our results point that fibrocytes could be a pathogenic link between joint and RA lung pathology. We found a moderate overall correlation between a decline in FVC and $\mathrm{DLCO}_{c}$ levels and an increased number of synovial fibrocytes. Fibrocytes have been found in higher levels in both circulation and lung biopsies in patients with Idiopathic Pulmonary Fibrosis (IPF) and ILD. ${ }^{12} 1335$ Hence, circulating fibrocytes have been proposed to be one of the sources of the expanding lung resident fibrocyte population and have been shown to be a marker of IPF disease activity. ${ }^{12}$ We find a weak correlation between the absolute levels of both circulating and synovial fibrocytes with the diffusion capacity. In contrast, only levels of synovial fibrocytes were moderately correlated with the changes in diffusion capacity during the trial period. Further, we found higher levels of circulating fibrocytes in the group with reduced diffusion capacity at 6 months compared with RA patients with normal diffusion capacity, indicating that fibrocytes could be involved in RA lung pathology. However, based on this small exploratory study, it cannot be concluded which of the two fibrocyte measurements is the strongest biomarker for the reduction in diffusion capacity in patients with RA.

Recently, drugs with strong fibrocyte inhibitor effects such as tyrosine kinase inhibition and a new drug based on the SAP protein have both shown an effect in severe idiopathic pulmonary fibrosis. ${ }^{144243}$ Studies are warranted to investigate if the new therapies targeting fibrocyte differentiation/migration could be a path forward in RA/RA-ILD treatment.

\section{Author affiliations}

${ }^{1}$ Department of Rheumatology, Odense Universitetshospital, Odense, Denmark ${ }^{2}$ Section of Rheumatology, Department of Medicine, Svendborg Hospital, Odense University Hospital, Svendborg, Denmark

${ }^{3}$ Department of Clinical Immunology, Odense University Hospital, Odense, Denmark ${ }^{4}$ Department of Orthopaedic Surgery, Odense University Hospital, Odense, Denmark ${ }^{5}$ Mental Health Services, Region of Southern Denmark, Odense, Denmark ${ }^{6}$ Department of Pathology, Odense University Hospital, Odense, Denmark ${ }^{7}$ Department of Radiology, Odense University Hospital, Odense, Denmark ${ }^{8}$ Centre for Experimental Medicine and Rheumatology, Barts and The London NHS Trust, London, UK

Acknowledgements We are indebted to all the study subjects and personnel at OUH contributing data to this study. A special thank you to Kasper Tholstrup Pedersen and the Department of Nuclear Medicine, Odense University Hospital for assisting with lung function tests.

Contributors All authors have contributed substantially in the process of completing this study, specified as follows. Conception of the study: SAJ, HL. Designing the study: SAJ, HL, CN. Aggregation of data: SAJ, CN, JCW, PVL, EKH, HT, HDS, TB, TT, FH, HL. Statistics: SAJ, PVL. Interpretation of data: All authors. Drafting and revising, final approval and agreement to be accountable: All authors.

Funding SAJ is supported by grants from The Danish Rheumatism Association and Odense University Hospital PhD Fund and fund for clinical research.

Competing interests None declared.

Patient consent for publication Not required.

Ethics approval The SynRA study is approved by the regional ethics review board (S-20140062) and the Danish Data Protection Agency (2008-58-0035).

Provenance and peer review Not commissioned; externally peer reviewed.

Data availability statement Data are available upon reasonable request. The datasets used and analysed during the current study are available from the corresponding author on reasonable request.

Open access This is an open access article distributed in accordance with the Creative Commons Attribution Non Commercial (CC BY-NC 4.0) license, which permits others to distribute, remix, adapt, build upon this work non-commercially, and license their derivative works on different terms, provided the original work is properly cited, appropriate credit is given, any changes made indicated, and the use is non-commercial. See: http://creativecommons.org/licenses/by-nc/4.0/.

\section{ORCID iDs}

Søren Andreas Just http://orcid.org/0000-0002-3946-5919

Helene Broch Tenstad http://orcid.org/0000-0002-3403-9865

\section{REFERENCES}

1 Herzog EL, Bucala R. Fibrocytes in health and disease. Exp Hematol 2010;38:548-56.

2 Galligan CL, Fish EN. Circulating fibrocytes contribute to the pathogenesis of collagen antibody-induced arthritis. Arthritis Rheum 2012;64:3583-93.

3 Galligan CL, Siminovitch KA, Keystone EC, et al. Fibrocyte activation in rheumatoid arthritis. Rheumatology 2010;49:640-51.

4 Bucala R. Fibrocytes in rheumatoid pannus: seed and soil? Arthritis Rheum 2012;64:3491-3. 
5 Lefèvre S, Knedla A, Tennie C, et al. Synovial fibroblasts spread rheumatoid arthritis to unaffected joints. Nat Med 2009;15:1414-20.

6 Lefevre S, Meier FMP, Neumann E, et al. Role of synovial fibroblasts in rheumatoid arthritis. Curr Pharm Des 2015;21:130-41.

7 Chong SG, Sato S, Kolb M, et al. Fibrocytes and fibroblasts-Where are we now. Int J Biochem Cell Biol 2019;116:105595.

8 Galligan CL, Keystone EC, Fish EN. Fibrocyte and T cell interactions promote disease pathogenesis in rheumatoid arthritis. $J$ Autoimmun 2016;69:38-50.

9 Nikam VS, Schermuly RT, Dumitrascu R, et al. Treprostinil inhibits the recruitment of bone marrow-derived circulating fibrocytes in chronic hypoxic pulmonary hypertension. Eur Respir J 2010;36:1302-14.

10 Kawano H, Koyama K, Nishimura H, et al. Development of improved method to identify and analyze lung fibrocytes with flow cytometry in a reporter mouse strain. Immun Inflamm Dis 2021:9:120-7.

11 Pilling D, Vakil V, Cox N, et al. TNF- $\alpha$-stimulated fibroblasts secrete lumican to promote fibrocyte differentiation. Proc Natl Acad Sci U S A 2015:112:11929-34.

12 Heukels P, van Hulst JAC, van Nimwegen M, et al. Fibrocytes are increased in lung and peripheral blood of patients with idiopathic pulmonary fibrosis. Respir Res 2018;19:90.

13 Fujiwara A, Kobayashi H, Masuya M, et al. Correlation between circulating fibrocytes, and activity and progression of interstitial lung diseases. Respirology 2012;17:693-8.

14 Raghu G, van den Blink B, Hamblin MJ, et al. Effect of recombinant human pentraxin 2 vs placebo on change in forced vital capacity in patients with idiopathic pulmonary fibrosis: a randomized clinical trial. JAMA 2018;319:2299-307.

15 Spagnolo $\mathrm{P}$, Lee JS, Sverzellati N, et al. The lung in rheumatoid arthritis: focus on interstitial lung disease. Arthritis Rheumatol 2018:70:1544-54

16 Peng H, Herzog EL. Fibrocytes: emerging effector cells in chronic inflammation. Curr Opin Pharmacol 2012;12:491-6.

17 Phillips RJ, Burdick MD, Hong K, et al. Circulating fibrocytes traffic to the lungs in response to CXCL12 and mediate fibrosis. J Clin Invest 2004;114:438-46.

18 Frid MG, Brunetti JA, Burke DL, et al. Hypoxia-Induced pulmonary vascular remodeling requires recruitment of circulating mesenchymal precursors of a monocyte/macrophage lineage. Am J Pathol 2006;168:659-69.

19 Just SA, Lindegaard H, Hejbøl EK, et al. Fibrocyte measurement in peripheral blood correlates with number of cultured mature fibrocytes in vitro and is a potential biomarker for interstitial lung disease in rheumatoid arthritis. Respir Res 2017;18:141.

20 Just SA, Nielsen C, Werlinrud JC, et al. Six-Month prospective tria in early and long-standing rheumatoid arthritis: evaluating disease activity in the wrist through sequential synovial histopathological analysis, RAMRIS magnetic resonance score and EULAR-OMERACT ultrasound score. RMD Open 2019;5:e000951.

21 Rheumatology TDSo. National treatment Guidlines for rheumatoid arthritis. Available: https://danskreumatologi.dk/nbv/sygdomme/ra/

22 Kelly S, Humby F, Filer A, et al. Ultrasound-Guided synovial biopsy: a safe, well-tolerated and reliable technique for obtaining high-quality synovial tissue from both large and small joints in early arthritis patients. Ann Rheum Dis 2015;74:611-7.

23 Krenn V, Perino G, Rüther W, et al. 15 years of the histopathological synovitis score, further development and review: a diagnostic score for rheumatology and orthopaedics. Pathol Res Pract 2017;213:874-81.
24 Pilling D, Gomer RH. Differentiation of circulating monocytes into fibroblast-like cells. Methods Mol Biol 2012;904:191-206.

25 Shao DD, Suresh R, Vakil V, et al. Pivotal advance: Th-1 cytokines inhibit, and Th-2 cytokines promote fibrocyte differentiation. $J$ Leukoc Biol 2008;83:1323-33.

26 van der Loos CM. Multiple immunoenzyme staining: methods and visualizations for the observation with spectral imaging. J Histochem Cytochem 2008;56:313-28.

27 Nihlberg K, Larsen K, Hultgårdh-Nilsson A, et al. Tissue fibrocytes in patients with mild asthma: a possible link to thickness of reticular basement membrane? Respir Res 2006;7:50.

28 Abu El-Asrar AM, Struyf S, Van Damme J, et al. Circulating fibrocytes contribute to the myofibroblast population in proliferative vitreoretinopathy epiretinal membranes. Br J Ophthalmol 2008;92:699-704.

29 Larsen A. How to apply Larsen score in evaluating radiographs of rheumatoid arthritis in long-term studies. $J$ Rheumatol 1995;22:1974-5.

30 Pellegrino R, Viegi G, Brusasco V, et al. Interpretative strategies for lung function tests. Eur Respir J 2005;26:948-68.

31 Stanojevic S, Graham BL, Cooper BG, et al. Official ERS technical standards: global lung function initiative reference values for the carbon monoxide transfer factor for Caucasians. European Respiratory Journal 2017;50:1700010.

32 Alhamad EH, Shakoor Z, Al-Kassimi FA, et al. Rapid detection of circulating fibrocytes by flowcytometry in idiopathic pulmonary fibrosis. Ann Thorac Med 2015;10:279-83.

33 Bianchetti L, Isgrò M, Marini MA, et al. Enumeration of circulating fibrocytes for clinical use in asthma by an optimized single-platform flow cytometry assay. BBA Clin 2014;1:52-8.

$34 \mathrm{Hu}$ X, DeBiasi EM, Herzog EL. Flow cytometric identification of fibrocytes in the human circulation. Methods Mol Biol 2015;1343:19-33.

35 Andersson-Sjöland A, de Alba CG, Nihlberg K, et al. Fibrocytes are a potential source of lung fibroblasts in idiopathic pulmonary fibrosis. Int J Biochem Cell Biol 2008;40:2129-40.

36 Schmidt M, Sun G, Stacey MA, et al. Identification of circulating fibrocytes as precursors of bronchial myofibroblasts in asthma. $J$ Immunol 2003;171:380-9.

37 Mori L, Bellini A, Stacey MA, et al. Fibrocytes contribute to the myofibroblast population in wounded skin and originate from the bone marrow. Exp Cell Res 2005;304:81-90.

38 Alivernini S, MacDonald L, Elmesmari A, et al. Distinct synovial tissue macrophage subsets regulate inflammation and remission in rheumatoid arthritis. Nat Med 2020;26:1295-306.

39 Orange DE, Yao V, Sawicka K, et al. Rna identification of prime cells predicting rheumatoid arthritis flares. N Engl J Med 2020;383:218-28.

40 Mizoguchi F, Slowikowski K, Wei K, et al. Functionally distinct disease-associated fibroblast subsets in rheumatoid arthritis. Nat Commun 2018;9:789.

41 Maharaj S, Shimbori C, Kolb M. Fibrocytes in pulmonary fibrosis: a brief synopsis. Eur Respir Rev 2013;22:552-7.

42 Flaherty KR, Wells AU, Cottin V, et al. Nintedanib in progressive fibrosing interstitial lung diseases. N Engl J Med 2019;381:1718-27.

43 Sato S, Shinohara S, Hayashi S, et al. Anti-fibrotic efficacy of nintedanib in pulmonary fibrosis via the inhibition of fibrocyte activity. Respir Res 2017;18:172. 